\title{
An experimental examination of fiber reinforcing effect with a novel composite specimen
}

\author{
Yuping Zhanga , Xungai Wang ${ }^{\mathrm{a}}$, Ning Pan ${ }^{\mathrm{b}, *}$ \\ ${ }^{a}$ School of Engineering and Technology, Deakin University, Geelong 3216, Australia \\ ${ }^{\mathrm{b}}$ Division of Textiles and Clothing, Department of Biological and Agricultural Engineering, University of California, Davis, CA 95616, USA
}

Received 14 April 2002; accepted 26 August 2002

\begin{abstract}
This paper introduces a simple fiber/tape composite that is easy to prepare and structurally more uniform. This novel composite provides a good alternative standard composite sample for experimental study of various phenomena and issues in fiber reinforced composite behaviors. Experimental results in the present work demonstrate how the fiber fragmentation, fiber-reinforcing effect and the gauge length effect can be successfully evaluated by using this novel composite specimen.

(C) 2003 Elsevier Science Ltd. All rights reserved.
\end{abstract}

Keywords: Novel standard composite specimen; B. Fragmentation; C. Micro-mechanics; E. Tape

\section{Introduction}

Fiber reinforced composites are increasingly utilized in aerospace, automotive, construction and other industries, because of their high strength/weight ratio and impact resistance as well as other distinctive attributes. Numerous research works have been carried out on composites reinforced with brittle fibers such as glass, carbon, Kevlar, boron and ceramic fibers. The research focus in this field has been on the prediction of the properties of a composite, for instance the tensile strength. The 'Rule of Mixtures' is the simplest model, which suggests the strength of a longitudinal continuous fiber reinforced composite is the volume fraction sum of the strengths of its constituents, assuming that all fibers have identical properties, and both fibers and matrix have the same breaking strain. This rule is obviously an oversimplification, because among other factors the properties of a composite are determined not only by the properties of its constituents but also by their distributions and the complex interactions between them. Parratt [1] among the earliest ones observed that fiber strength is not uniform and the reinforcing fibers can break into many fragments until the load transferred from the fiber/matrix interface is not enough to break the fragments any more

\footnotetext{
* Corresponding author.

E-mail address: npan@ucdavis.edu (N. Pan).
}

(known as the saturation state). By considering that the fiber breaks are caused by the statistically distributed flaws, Rosen [2] established a model that treats the load redistribution among the unbroken fibers after a fiber break as equal. Later, Zweben [3] considered the load redistribution as local and verified that the local load sharing model can predict the break load of a composite more accurately.

According to the weakest-link theory [4], fibers can be treated as being made of many connecting segments whose properties are statistically distributed, and they always break in their weakest point. Weibull distribution is widely applied to strength of fibers for fiber reinforced composites. It was found that the Weibull parameters obtained by single fiber test were in the same range as that by single fiber composite test [5-8]. But the in situ fiber behaviors in fiber composite are quite different from those of single fibers or fiber bundles, because the broken fibers in composite experience interactions between fibers and the matrix and among fibers themselves; the result of the interactions manifests itself in the form of the fragmentation process. This fiber fragmentation process is understandably affected by the applied stress, the in situ fiber properties and the fiber/ matrix interactions.

In order to examine the failure mode of the composite, Rosen [2] utilized a composite specimen with a single layer of glass fibers embedded in an epoxy. The in situ fiber stress 
distribution (the stressed and the unstressed area) and the ineffective fiber length can be observed clearly by using the polarized transmitted light when stretching the specimen. Later the similar but dog-bone shaped single fiber composite test has been widely used to study the in situ fiber properties and fiber/matrix interfacial properties [5-15]. By monitoring the fiber fragmentation optically or acoustically, the distribution of fiber fragment length during the testing and at the saturation can be obtained [26,27]. Recently, Park et al. [16] used a multi-fiber specimen with gradual difference spacing between individual fibers to study the inter-fiber interaction and the effects of the inter-fiber spacing.

The procedures of making such specimens usually involve the following steps [7]:

- mixing the epoxy resin with the curing agent with thorough stirring to remove the air;

- pouring the mixture into a rubber mould in which a single fiber is suspended in the centre;

- putting the mould containing the fiber and the epoxy into the oven to cure at $80{ }^{\circ} \mathrm{C}$ for $3 \mathrm{~h}$;

- turning off the oven and cooling the specimen slowly to reduce the residual stresses;

- polishing the specimen.

Extreme care is necessary during specimen preparation so that no air get into the mixture and the fiber inside the mould is not disturbed when pouring the matrix mixture into the mould. Still, the composite specimens made this way are notoriously known to be highly prone to gross structural non-uniformity and property variations.

The significance of having standard composite specimens (or micro-composites) was elaborated by Wagner et al. [17]. Their composite specimens are thin films of polymeric matrix with single fibers accurately positioned inside. As stated by Wagner et al. [17], the experimental standard composite specimens can be used to verify the theoretical models on composite fracture, since the parameters of this composite specimen can be controlled close to those of the theoretical model. Furthermore, other issues such as stress redistribution upon initial fiber breakage, fiber/matrix interaction, in situ fiber properties, as well as the influence of fiber spacing on composite failure in a multi-fiber composite can also be investigated. A special fiber positioning technique was developed $[18,19]$ when making this micro-composite. However, even with the improvement, the variations among the composite specimens thus prepared are still large due to the nature of the delicate and tedious specimen preparation process. This grossly impedes the research efforts in composites.

In this work, a much simpler alternative way to make such composite specimens is introduced. This novel microcomposite thus prepared is much more uniform because of the easy control of the specimen preparation process and hence is inherently more reliable to be used to examine the fiber reinforcing effect on the break load, strength and break strain of the composite as presented in this study. The fiber fragmentation and the contribution of the reinforcing fiber to the behavior of composite are also investigated using this method.

\section{The novel composite specimen}

This simple composite specimen is prepared by aligning and sandwiching single or multiple fibers between two single-sided adhesive tapes. The transparent tapes and black fibers are used in this work for easy observation of the fiber fragmentation process. To facilitate the occurrence of the fiber fragmentation process, the breaking strain of the tape is nearly two times of that of the fibers at gauge length $100 \mathrm{~mm}$.

Before placing the fibers in the tapes, each fiber was scanned at $40 \mu \mathrm{m}$ interval for more accurate diameter measurement along fiber length using a single fiber analyser SIFAN [20], and tested for the tensile properties on an Instron Tester 1122 according to ASTM D1445-90.

The tensile properties of the tapes were tested on the same machine based on ASTM D3822-02; and for easy comparison, we used the sample formed by tape of two layers adhered together, i.e. a similar sample as above but with no reinforcing fiber. The property uniformity of the tape can be inferred from the relatively small CV\% of the tensile test results (Tape only, Table 1).

With this technique, several sets of composite specimens were prepared, using cellophane ${ }^{\circledR}$ tapes as the matrix and the black dyed wool as the reinforcing fibers. The tape is $0.12 \mathrm{~mm}$ thick and $18 \mathrm{~mm}$ wide according to both the specifications and our verification,

For consistence, each specimen was prepared the following way:

1. Carefully mount the bottom layer of the tape on to a plane where the position and gauge length were preset by two adhesive strips accurately spaced and secured as shown in Fig. 1(a); with the sticky side up;

2. Hold a single fiber at both ends, adhere one end to the location tape and leave the other end free as in Fig. 1(b);

3. To attach a small weight determined according to ASTM D1445-90 to provide a proper pretension in the fiber, and then adhere the other end of the fiber to the position strip at the bottom as in Fig. 1(b);

4. Cover the top layer of the tape as in Fig. 1(c) and secure both the fiber ends;

Table 1

The mean fragment lengths for 1-fiber/tape specimen

\begin{tabular}{lc}
\hline Gauge length $(\mathrm{mm})$ & Mean fragment length $(\mathrm{mm})$ \\
\hline 20 & 7.02 \\
50 & 19.39 \\
100 & 22.95 \\
\hline
\end{tabular}




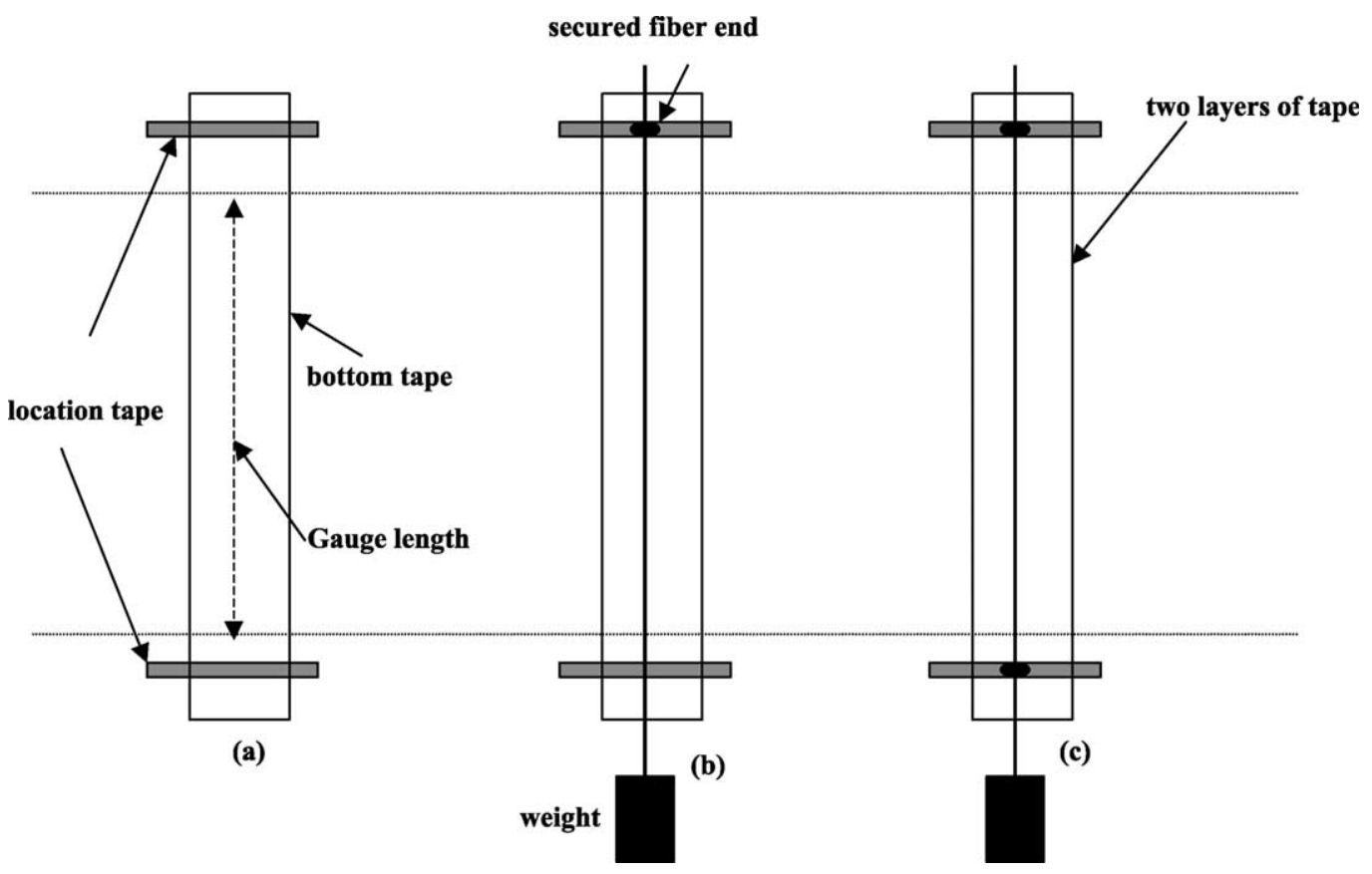

Fig. 1. The illustration of the specimen preparation.

5. Using a steel ruler, press the specimen to assure complete adhesion between the fiber-tape and tape-tape;

6. After carefully examining the bonding uniformity within and among all the specimens, cure and cut the specimen into size.

Both 1-fiber/tape and 3-fiber/tape composite specimens were prepared. All specimens have approximately the same thickness of $0.12 \mathrm{~mm}$, as measured on a Shirley Thickness Gauge. The average fiber volume fraction is 0.03 for 1fiber/tape and 0.09 for 3-fiber/tape specimens.

After examining the specimen quality under magnifying glasses, the tensile properties of the specimens were tested on an Instron at several gauge lengths and a crosshead speed of $20 \mathrm{~mm} / \mathrm{min}$.

\section{Results and discussion}

\subsection{Fiber fragmentation}

It is widely known that in a fiber reinforced composite, if the breaking strain of the matrix is significantly greater than that of the reinforcing fibers, the fibers inside the matrix will break first when reaching its breaking strain. The fibers will continue to break until the fragment length reaches the socalled critical or saturation length when the load transferred from the matrix cannot be built up to break the fiber any more, leading to eventual failure of the composite.

As shown in Fig. 2, fiber fragmentation can be clearly observed by means of this new method, and the fragment length is not uniform but randomly distributed. The distribution of fiber fragment length has been studied before
$[6,7,21,22]$. This scatter in fragment length can be easily understood as due to the property variations along the fiber length. For this novel specimen, we can largely eliminate some of the error sources such as the fiber damage and local disorientation during resin pouring, and irregular interface due to existing temperature or humidity gradient and uneven resin fiber contact often associated with the specimens prepared in conventional way.

The average fragment lengths for the 1-fiber/tape specimen at the end of the test were measured and they are found different for samples measured under different gauge lengths as shown in Table 1. It can be seen that the average fragment length increases with the increasing gauge length, seemingly suggesting that the interfacial stress transfer between the reinforcing fiber and the matrix is less effective when the gauge length is longer. This may be explained from previous theoretical and experimental works. Firstly, the tensile stress on fiber fragments transferred from the matrix is proportional to the applied stress on composite specimen according to Cox's shear-lag theory [23] and other model [24]. Secondly, it is observed experimentally that the average fiber fragment length is decreasing with the increasing applied stress before the saturation state of fiber

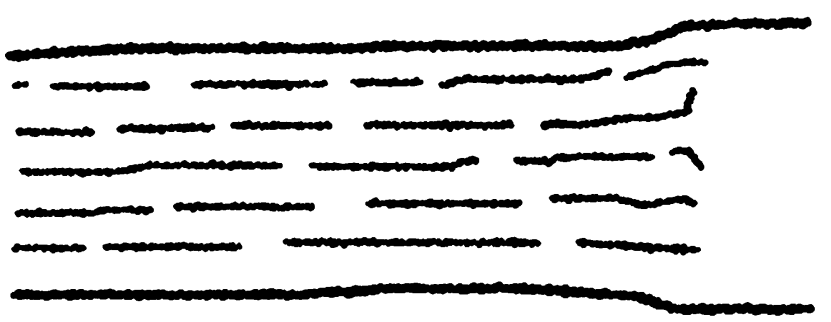

Fig. 2. A photo showing fragmented fibers sandwiched in tapes. 
Table 2

The break loads of single fibers, tape only and fiber/tape

\begin{tabular}{|c|c|c|c|c|c|c|c|c|c|c|c|c|}
\hline \multirow[t]{2}{*}{ GL } & \multicolumn{3}{|l|}{ Single fiber } & \multicolumn{3}{|l|}{ Tape only } & \multicolumn{3}{|c|}{ 1-fiber/tape } & \multicolumn{3}{|c|}{ 3-fiber/tape } \\
\hline & Mean $(\mathrm{mN})$ & $\mathrm{CV}(\mathrm{mN})$ & $n$ & Mean $(\mathrm{N})$ & $\mathrm{CV}(\mathrm{N})$ & $n$ & Mean $(\mathrm{N})$ & $\mathrm{CV}(\mathrm{N})$ & $n$ & Mean $(\mathrm{N})$ & $\mathrm{CV}(\mathrm{N})$ & $n$ \\
\hline 20 & 62.49 & 43.62 & 153 & 141.18 & 9.83 & 25 & 143.84 & 3.47 & 13 & 145.70 & 2.75 & 17 \\
\hline 50 & 50.49 & 32.60 & 37 & 140.34 & 4.01 & 33 & 140.35 & 4.99 & 29 & 141.90 & 4.58 & 30 \\
\hline 100 & 49.00 & 36.43 & 51 & 134.50 & 3.94 & 28 & 129.33 & 5.83 & 20 & 132.70 & 3.35 & 29 \\
\hline
\end{tabular}

GL: gauge length (mm); $n$ : sample number.

fragmentation [13,24]. According to the weakest link theory, the strength of the composite specimens is lower at the longer gauge length. So it could be inferred that the average fiber fragment length at saturation should be smaller at a shorter gauge length due to the higher stress in the fibers.

\subsection{Break load}

The results of the break load for the specimens of single fibers, fiber/tape and tape without fibers are summarised in Table 2. Assuming the reinforcing fibers are uniform in their properties and diameters and a perfect bonding exists, the load sustained by the composite should be shared by the fibers and the matrix in the initial stage of the extension as

$P_{\mathrm{c}}=P_{\mathrm{f}}+P_{\mathrm{m}}$,

where the subscripts $\mathrm{c}, \mathrm{f}$ and $\mathrm{m}$ represent composite, fiber and matrix, respectively.

Assuming the area fractions equal to volume fractions, Eq. (1) can be readily converted into the well-known Rule of Mixtures:

$\sigma_{\mathrm{c}}=\sigma_{\mathrm{f}} V_{\mathrm{f}}+\sigma_{\mathrm{m}} V_{\mathrm{m}}$,

where $\sigma$ is the stress and $V$ is the volume fraction. If such a simple model as Eq. (1) for the average breaking load of

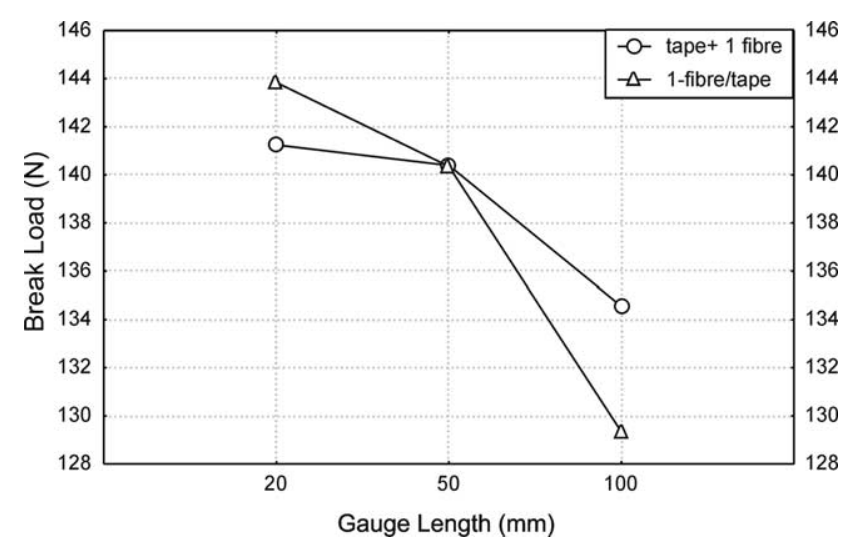

Fig. 3. Comparison of the average break load of 1-fiber/tape with the sum of that of tape and one single fiber. the fiber/tape system is valid, we should have:

$\overline{\mathrm{BF}_{1 \mathrm{f} / \mathrm{t}}}=\overline{\mathrm{BF}_{\mathrm{f}}}+\overline{\mathrm{BF}_{\mathrm{t}}}$,

and

$\overline{\mathrm{BF}_{3 \mathrm{f} / \mathrm{t}}}=3 \overline{\mathrm{BF}_{\mathrm{f}}}+\overline{\mathrm{BF}_{\mathrm{t}}}$,

where $\overline{\mathrm{BF}}$ is the mean breaking load, subscripts $1 \mathrm{f} / \mathrm{t}, 3 \mathrm{f} / \mathrm{t}$, $\mathrm{f}$ and $t$ represent 1-fiber/tape composite, 3-fiber/tape composite, single fiber, and tape without fiber, respectively.

According to the experiments, however, the average break loads in Eqs. (3) and (4) of fiber/tape (1-fiber/tape and 3 -fiber/tape) and the sums of that of single fibers and tapes without fiber are compared and shown in Fig. 3 for 1-fiber/ tape and in Fig. 4 for 3-fiber/tape.

Figs. 3 and 4 indicate a well known fact that the simple Rule of Mixtures is in general not applicable to the breaking load of a composite. Since the matrix has a much higher breaking strain and fibers are variable in their diameters and properties among fibers and along a fiber, the weak point in a fiber breaks first when the composite is under the tension. This fiber breakage will cause high stress concentration (static and dynamic) in its surrounding area that may lead to the de-bonding around the broken fiber end. On the other hand, broken fibers in the composite can sustain the load again and thus cause the fiber fragmentation with each fragment being further broken before saturation. As a result,

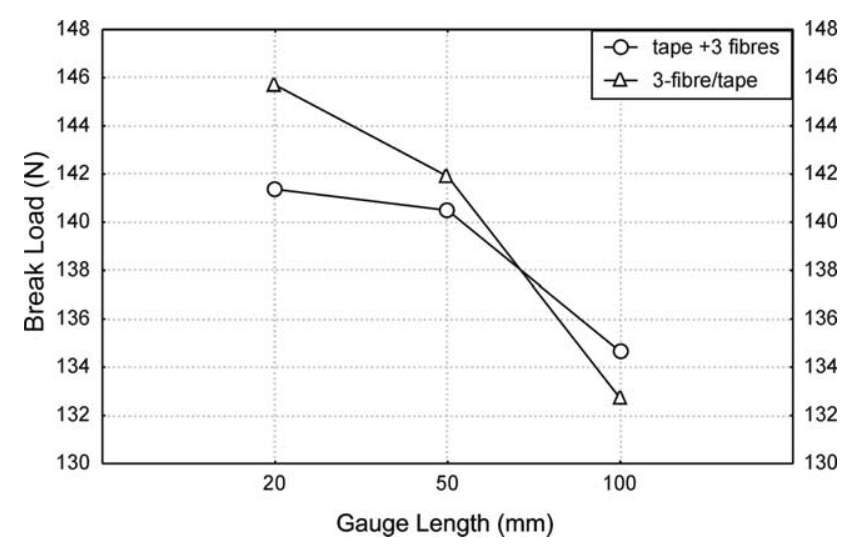

Fig. 4. Comparison of the average break load of 3-fiber/tape with the sum of that of two single fibers and tape. 
Table 3

The strengths of single fibers, tape only and fiber/tape system

\begin{tabular}{|c|c|c|c|c|c|c|c|c|c|c|c|c|}
\hline \multirow[t]{2}{*}{ GL } & \multicolumn{3}{|l|}{ Single fiber } & \multicolumn{3}{|l|}{ Tape only } & \multicolumn{3}{|l|}{ 1-fiber/tape } & \multicolumn{3}{|l|}{ 3-fiber/tape } \\
\hline & Mean (MPa) & $\mathrm{CV}(\mathrm{MPa})$ & $n$ & Mean (MPa) & $\mathrm{CV}(\mathrm{MPa})$ & $n$ & Mean (MPa) & $\mathrm{CV}(\mathrm{MPa})$ & $n$ & Mean (MPa) & $\mathrm{CV}(\mathrm{MPa})$ & $n$ \\
\hline 20 & 213.44 & 17.84 & 153 & 65.59 & 10.00 & 25 & 66.59 & 3.47 & 13 & 67.47 & 2.75 & 17 \\
\hline 50 & 205.38 & 14.02 & 37 & 64.97 & 4.01 & 33 & 64.98 & 4.99 & 29 & 65.71 & 4.58 & 30 \\
\hline 100 & 201.36 & 18.28 & 51 & 62.27 & 3.94 & 28 & 59.88 & 5.83 & 20 & 61.43 & 3.35 & 29 \\
\hline
\end{tabular}

GL: gauge length $(\mathrm{mm}) ; n$ : sample number.

the final fracture of the composite specimen should be resulted from the combination of the above sophisticated mechanisms and thus its break load should not be just the simple sum of those of its two constituents.

It is also observed from both Figs. 3 and 4 that the fibertape interactions in the specimen might play a different role at different gauge lengths. For specimens at short gauge length $(20 \mathrm{~mm})$, the high fiber-matrix interactions lead to more saturated fiber fragmentation of shorter critical length, which brings about a positive synergistic effect, whereas for specimens at $100 \mathrm{~mm}$ gauge length, the cumulative weakening and the high local stress concentration (static and dynamic) resulting from the fiber breakage seem to have a dominant effect. It appears reaching a balance between these two competing effects for specimens with the gauge length of $50 \mathrm{~mm}$.

For a multi-fiber composite, the stress is shared by more than one fiber, and it will be redistributed in the vicinity after a fiber break. The adjacent fibers can block the cracks caused by this breakage. These leads to the different extent of the fiber reinforcing or weakening effect for 3-fiber/tape from that of 1-fiber/tape model composite as shown clearly in Figs. 3 and 4.

\subsection{Strength}

The tensile strengths of all specimens tested are calculated and summarised in Table 3 . In order to examine the contribution of the reinforcing fiber to the strength of the fiber/tape composite at the present low fiber volume fractions, the strength of tape without any fibers is compared with that of 1-fiber/tape and 3-fiber/tape composites. This is shown in Fig. 5.

Fig. 5 clearly demonstrates that although fiber volume fraction is extremely low in these specimens, fiber/tape composite is still stronger than the tape for those measured at short gauge lengths $(20 \mathrm{~mm})$. However, this is not the case at the gauge length of $100 \mathrm{~mm}$. This might again be explained from the average fiber fragment lengths as shown in Table 1. The mean fragment length is longer at a greater gauge length, meaning the load which can be transferred from the tape to the fiber is small at long gauge length. Because of the limited load transfer, the fiber strength cannot be effectively realized at the long gauge length. In contrast, the cumulative weakening caused by fiber breakage and the local stress concentrations has a negative effect on the composite. Fig. 5 also exhibits as expected that the strength generally increases with the increasing fiber volume fraction, i.e. the strength, of 3-fiber/tape system is always higher than that of 1-fiber/tape system. In other words, a high fiber volume fraction is needed in order to obtain the positive synergistic effect of the composite, particularly at long gauge length.

\subsection{Breaking strain}

Table 4 summarises the results of the break strains for single fibers, tape without fibers and fiber/tape specimens. The break strains of fiber/tape specimens at three gauge lengths are again compared with those of tape without any fibers as shown in Fig. 6. Fig. 6 indicates that similar trends to the strength exist for the break straining as well. The reinforcing fibers have either no or only marginally negative effects on the break strain of the composite when the gauge length is long (50 and $100 \mathrm{~mm}$ ). However, the break strain of the fiber/tape specimen at $20 \mathrm{~mm}$ gauge length does increase. The fiber volume fraction appears to have an

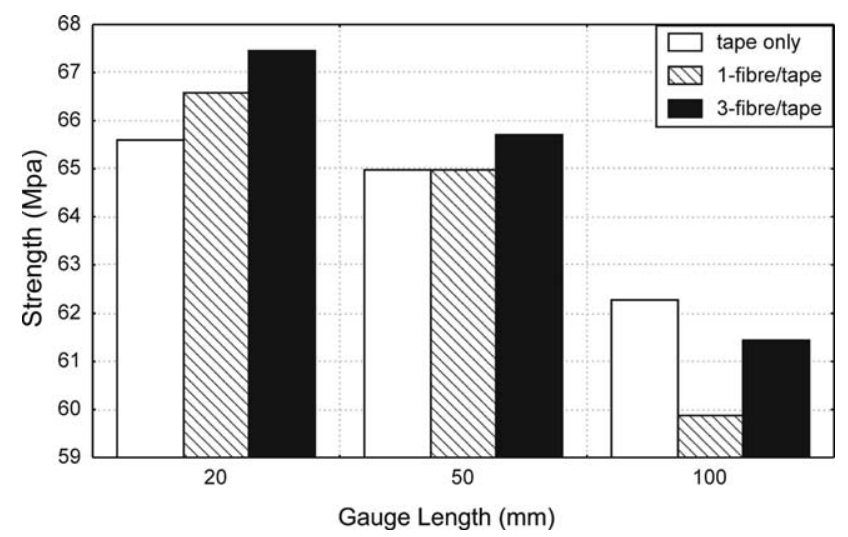

Fig. 5. The strength of fiber/tape composite compared with that of tape without any fibers. 
Table 4

The break strains of single fibers, tape only and fiber/tape specimen

\begin{tabular}{|c|c|c|c|c|c|c|c|c|c|c|c|c|}
\hline \multirow[t]{2}{*}{ GL } & \multicolumn{3}{|l|}{ Single fiber } & \multicolumn{3}{|l|}{ Tape only } & \multicolumn{3}{|l|}{ 1-fiber/tape } & \multicolumn{3}{|l|}{ 3-fiber/tape } \\
\hline & Mean $(\%)$ & $\mathrm{CV}(\%)$ & $n$ & Mean $(\%)$ & $\mathrm{CV}(\%)$ & $n$ & Mean $(\%)$ & $\mathrm{CV}(\%)$ & $n$ & Mean $(\%)$ & $\mathrm{CV}(\%)$ & $n$ \\
\hline 20 & 37.10 & 37.36 & 153 & 50.86 & 8.29 & 25 & 52.95 & 5.58 & 13 & 53.53 & 4.72 & 17 \\
\hline 50 & 25.57 & 44.00 & 37 & 42.09 & 5.97 & 33 & 39.07 & 8.36 & 29 & 39.83 & 7.40 & 30 \\
\hline 100 & 19.06 & 49.37 & 51 & 38.39 & 5.84 & 28 & 37.72 & 8.63 & 20 & 38.59 & 5.06 & 29 \\
\hline
\end{tabular}

GL: gauge length (mm); $n$ : sample number.

augmenting effect on the break strain of the model composite as in the case for the strength.

\subsection{Gauge length effect}

The data in this study show that the break load, the strength and the break strain of single fibers, tape without fiber and fiber/tape specimens all decrease with the increasing gauge length. These results also indicate, as discussed above, that the gauge length not only influences the tensile behavior of the materials but also the efficiency of the fiber reinforcement, the fiber/matrix interactions, as well as the inter-fiber interactions in multi-fiber composite.

It has to be pointed out here that the different strain rate is actually applied in the tests at the different gauge lengths, because the test speed is kept constant. This, however, should not invalidate this study [25], since all the new findings in this study obtained from comparisons made within the same gauge length; the potential effect of the strain rate is virtually eliminated.

The advantages of this new specimen can be further elucidated using the testing data in Tables 3 and 4. The standard deviations for both strength and strain are much large in case of single fiber than those of tape alone. This shows the inherent uniformity in tape properties. Then the data for the fiber/tape system are in the same order of

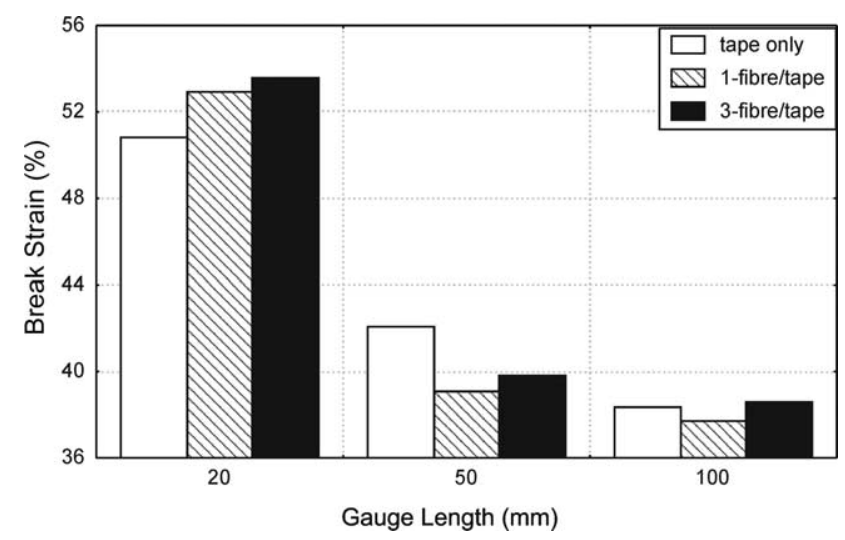

Fig. 6. The break strain of fiber/tape composite compared with that of tape without any fibers. magnitude as those of the tape, significantly smaller than those of the single fiber. This indicates that the specimens largely preserve the property uniformity of the tape; the influences of specimen preparation in deteriorating the specimen quality due to problems in the conventional practice can be largely avoided.

\section{Conclusions}

The following conclusions can be drawn from this study:

- The proposed fiber/tape micro-composite can be used as a simple yet structurally more uniform specimen in investigating various phenomena and issues in fiber reinforced composites. The fiber fragmentation can be clearly observed and the distribution of fiber fragments at the end of test can be easily obtained.

- The mean fragment length is longer at the long gauge length than that at the short gauge length, seemingly suggesting that the fiber-matrix interactions are greater when the gauge length is shorter.

- The break load of fiber/tape specimen has been shown as expected to be higher than the arithmetic sum of that of the fiber and tape at short gauge length $(20 \mathrm{~mm})$. A higher fiber volume fraction is needed to improve the fiber reinforcing effect, especially at long gauge lengths. This may provide to certain degree the validity of using the proposed specimen for composite research.

- The strength and the break strain of fiber/tape composite are enhanced compared with those of tape without fiber at a short gauge length. The behavior of the specimen, however, deteriorates at a long gauge length; poor load transfer at the long gauge length and the stress concentrations at the fiber ends might be responsible for this.

- The advantages of the proposed specimen can be reflected from the fact that after the composite specimen preparation, the variation in the system is no greater than that of either constituent. That is, the preparation process brings in negligible irregularities, which is the vital problem in existing practices. 


\section{References}

[1] Parratt NJ. Defects in glass fibers and their effect on the strength of plastic mouldings. Rubber Plastics Age 1960;March:263-6.

[2] Rosen BW. Tensile failure of fibrous composites. AIAA J 1964;2: 1985.

[3] Zweben C. Tensile failure of fiber composites. AIAA J 1968;6: 2325-31.

[4] Peirce FT. Tensile tests for cotton yarns - the Wealest link-theorems on the strength of long and of composite specimens. J Text Inst 1926; 17:T355-68.

[5] Baxevanakis C, Jeulin D, Valentin D. Fracture statistics of single-fiber composite specimens. Compos Sci Technol 1993;48:47-56.

[6] Andersons J, Tamuzs V. Fibre and interface strength distribution studies with the single-fiber composite test. Compos Sci Technol 1993;48:57-63.

[7] Curtin WA, Netravali AN, Park JM. Strength distribution of carborundum polycrystalline $\mathrm{SiC}$ fibres as derived from the single fiber composite test. J Mater Sci 1994;29:4718-28.

[8] Goda K, Park JM, Netravali AN. A new theory to obtain Weibull fiber strength parameters from a single fiber composite test. J Mater Sci 1995;30:2722.

[9] Henstenburg RB, Phoenix SL. Interfacial shear strength studies using the single-filament-composite test. Part2. A probability model and Monte Carlo simulation. Polym Compos 1989;10:389-408.

[10] Netravali AN, Henstenburg RB, Phoenix SL, Schwartz P. Interfacial shear strength studies using the single-filament-composite test. Part 1. Experiments on graphite fibers in epoxy. Polym Compos 1989;10: 226-41.

[11] Glushko VI, Kovalenko VP, Mileiko ST, Tvardovsky VV. Evaluation of fiber strength characteristics on the basis of the fiber fragmentation test. J Mater Sci 1993;28:6307-12.

[12] Shioya M, Takaku A. Estimation of fibre and interfacial shear strength by using a single-fiber composite. Compos Sci Technol 1995;55:33-9.

[13] Deng S, Ye L, Mai Y-W, Liu H-Y. Evaluation of fiber tensile strength and fiber/matrix adhesion using single fiber fragmentation tests. Composites, Part A 1998;29:423-34.
[14] Kim BW, Nairn JA. Experimental verification of the effects of friction and residual stress on the analysis of interfacial debonding and toughness in single fiber composites. J Mater Sci 2002;37: 3965-72.

[15] Yilmaz YI. Analysing single fiber fragmentation test data by using stress transfer model. J Compos Mater 2002;36:537-51.

[16] Park JM, Kim JW, Goda K. A new method of evaluating the interfacial properties of composites by means of the gradual multi-fiber fragmentation test. Compos Sci Technol 2000;60: 439-50.

[17] Wagner HD, Rubins M, Marom G. The significance of microcomposites as experimental models. Polym Compos 1991;12:233-6.

[18] Wagner HD, Steenbakkers LW. J Mater Sci 1989;24:3956.

[19] Zhou XF, Wagner HD, Nutt SR. Interfacial properties of polymer composites measured by push-out and fragmentation tests. Compos, Part A: Appl Sci Manufact 2001;32:1543-51.

[20] Peterson AD, Brims A, Brims MA, Gherardi SG. Measuring the diameter profile of single wool fibers by using the single fibre analyser. J Text Inst 1998;89:441-8.

[21] Curtin WA. Exact theory of fiber fragmentation in a single-filament composite. J Mater Sci 1991;26:5239-53.

[22] Dibenedetto AT, Gurvich MR. Statistical simulation of fiber fragmentation in a single-fiber composite. Compos Sci Technol 1997;57:543-55.

[23] Cox HL. The elasticity and strength of paper and other fibrous materials. Br J Appl Phys 1952;3:72-9.

[24] Zhou L, Kim J-K, Baillie C, Mai Y-W. Fracture mechanics analysis of the fiber fragmentation test. J Compos Mater 1995;29:881-902.

[25] Pan N, Chen HC, Thompson J, Inglesby MK, Khatua S, Zhang XS, Zeronian $\mathrm{SH}$. The size effects on the mechanical behaviour of fibers. J Mater Sci 1997;32:2677-85.

[26] Penning JP, De Vries AA, Van Der Ven J, Pennings AJ, Hoogstraten HW. A study of transverse and longitudinal size effects in high strength polyethylene fibers. Philos Mag A 1994;69:267-84.

[27] Schwartz P, Netravali A, Sembach S. Effects of strain rate and gauge length on the failure of ultra-high strength polyethylene fibers. Text Res J 1986;56:502. 